\title{
Adaptor protein Crkll negatively regulates osteoblast differentiation and function through JNK phosphorylation
}

\author{
Jung Ha Kim¹, Kabsun Kim, Inyoung Kim, Semun Seong ${ }^{1,2}$, Kwang-\| Nam³, Kyung Keun Kim and Nacksung Kim ${ }^{1,2}$
}

\begin{abstract}
The adaptor protein Crkll is involved in several biological activities, including mitogenesis, phagocytosis, and cytoskeleton reorganization. Previously, we demonstrated that Crkll plays an important role in osteoclast differentiation and function through Rac1 activation both in vitro and in vivo. In this study, we investigated whether Crkll also regulates the differentiation and function of another type of bone cells, osteoblasts. Overexpression of Crkll in primary osteoblasts inhibited bone morphogenetic protein (BMP) 2-induced osteoblast differentiation and function, whereas knockdown of Crkll expression exerted the opposite effect. Importantly, Crkll strongly enhanced c-Jun-N-terminal kinase (JNK) phosphorylation, and the Crkll overexpression-mediated attenuation of osteoblast differentiation and function was recovered by JNK inhibitor treatment. Furthermore, transgenic mice overexpressing Crkll under control of the alpha-1 type I collagen promoter exhibited a reduced bone mass phenotype. Together, these results indicate that Crkll negatively regulates osteoblast differentiation and function through JNK phosphorylation. Given that Crkll acts as a negative and positive regulator of osteoblast and osteoclast differentiation, respectively, the regulation of Crkll expression in bone cells may help to develop new strategies to enhance bone formation and inhibit bone resorption.
\end{abstract}

\section{Introduction}

Osteoblasts are mononuclear cells that are responsible for bone formation. The differentiation of mesenchymal cells into osteoblasts is regulated by various anabolic factors, such as bone morphogenetic proteins (BMPs), insulin, and insulin-like growth factor- $1^{1-3}$. Notably, BMPs, which belong to the transforming growth factor- $\beta$ (TGF- $\beta$ ) superfamily of proteins, were initially discovered to induce bone formation. Binding of BMPs to their type I and II serine/threonine kinase receptors activates the Smad signaling pathway, inducing the expression of various target genes, such as runt-related transcription factor 2 (Runx2), alkaline phosphatase (ALP), and osteocalcin ${ }^{4-6}$.

Correspondence: Nacksung Kim (nacksung@jnu.ac.kr)

'Department of Pharmacology, Chonnam National University Medical School, Gwangju 61469, Republic of Korea

${ }^{2}$ Department of Biomedical Sciences, Chonnam National University Medical School, Gwangju 61469, Republic of Korea

Full list of author information is available at the end of the article.
Various transcription factors involved in osteoblast differentiation and function, such as the Runx2, osterix, and homeodomain-containing Msx proteins, have been well studied ${ }^{1,7-10}$. In particular, Runx 2 is a master transcription factor that regulates the expression of many osteoblastic marker genes, including ALP, bone sialoprotein (BSP), and osteocalcin to induce osteoblast differentiation and bone formation ${ }^{11,12}$. Runx2, also known as Cbfa1, belongs to the runt homology domain protein family and contains a glutamine/alanine-rich domain at its $\mathrm{N}$-terminal end (a runt domain) and a proline/serine/ threonine-rich region at its C-terminus ${ }^{11}$. Runx2 has been shown to be an essential transcription factor for osteoblast differentiation and mineralization in multiple in vitro and in vivo studies. Mice with a homozygous mutation in Runx 2 exhibit a complete lack of bone formation due to ossification failure ${ }^{13,14}$. Furthermore, mice with a heterozygous mutation in Runx2 exhibit cleidocranial dysplasia disorders associated with defective 
endochondral and intramembranous bone formation ${ }^{13,15}$. In contrast, transgenic mice overexpressing Runx2 show an increase in mineralizing bone surface, mineral apposition rate, and bone formation. Furthermore, the ectopic expression of Runx2 in even nonosteoblastic cells induces the expression of major osteoblastic marker genes ${ }^{16}$.

Recently, mitogen-activated protein kinase (MAPK) cascades, including the c-Jun-N-terminal kinase (JNK), extracellular signal-regulated kinase (Erk), and p38 cascades, have been revealed as noncanonical pathways for BMP2 signal transduction ${ }^{17-20}$. MAPKs can control osteoblast differentiation through the regulation of Runx2 phosphorylation and transcriptional activity. Runx2 phosphorylation by both Erk and p38 promotes BMP2induced osteoblast differentiation ${ }^{21,22}$. In contrast, Runx2 phosphorylation by JNK inhibits osteoblast differentiation via the negative regulation of Runx 2 function ${ }^{6}$.

CrkII belongs to a family of adaptor proteins that includes CrkI, CrkII, CrkIII, and CrkL ${ }^{23}$. The Crk family adaptor proteins predominantly contain Src homology 2 (SH2) and $\mathrm{SH} 3$ protein-binding domains ${ }^{24,25}$. Notably, CrkII contains one N-terminal SH2 domain and two SH3 domains (nSH3 and $\mathrm{cSH} 3$ ). Although it lacks an enzymatic kinase domain, CrkII plays a central role in several biological activities, such as mitogenesis, phagocytosis, and cytoskeleton reorganization, through constructing distinct combinations of protein interactions ${ }^{26-29}$. In our previous study, we demonstrated that CrkII plays critical roles in osteoclast differentiation and function through the regulation of Rac1 activity ${ }^{23}$. Since CrkII is ubiquitously expressed in most tissues, in the present study, we investigated the potential role of CrkII in osteoblast differentiation and function to clarify its role in bone homeostasis.

\section{Materials and methods Osteoblast differentiation}

The Committee of Ethics in Animal Research approved all animal procedures performed in this study (approval number: CNU IACUC-H-2017-27). Primary osteoblast precursor cells were isolated from neonatal ICR mouse (Dae Han BioLink Co., Korea) skulls by enzymatic digestion using $0.1 \%$ collagenase (Life Technologies, Carlsbad, CA) and 0.2\% dispase II (Roche Diagnostics $\mathrm{GmbH}$, Mannheim, Germany). The cells were cultured in osteogenic medium (OGM) containing BMP2 (100 ng/ $\mathrm{mL})$, ascorbic acid $(50 \mu \mathrm{g} / \mathrm{mL})$, and $\beta$-glycerophosphate $(100 \mathrm{mM})$ for osteoblast differentiation. Osteoblast precursor cells cultured for 3 days were used for ALP activity analysis to evaluate osteoblast differentiation. Cells were lysed using an osteoblast lysis buffer $[50 \mathrm{mM}$ Tris- $\mathrm{HCl}$ (pH 7.4), $1 \%$ Triton X-100, $150 \mathrm{mM} \mathrm{NaCl}$, and $1 \mathrm{mM}$ EDTA]. Cell lysates were incubated with $p$-nitrophenyl phosphate substrate (Sigma-Aldrich, St Louis, MO), and
ALP activity was measured by measuring the absorbance at $405 \mathrm{~nm}$ using a spectrophotometer. To evaluate mineralization, cells were cultured for 9-12 days, fixed with $70 \%$ ethanol, and stained with $40 \mathrm{mM}$ alizarin red (pH 4.2). After nonspecific staining was removed with a phosphate-buffered saline wash, alizarin red staining was visualized with a CanoScan 4400F scanner (Canon Inc., Japan). To quantify substrate calcification, alizarin redstained cells were incubated with a $10 \%$ cetylpyridinium chloride solution for $30 \mathrm{~min}$ at room temperature, and the absorbance at $562 \mathrm{~nm}$ was measured.

\section{Retroviral gene transduction}

Retroviral vectors were transfected into the Plat-E packaging cell line using FuGENE 6 (Promega, Madison, WI) according to the manufacturer's protocol. Viral supernatants were recovered from the culture medium at $48 \mathrm{~h}$ after transfection. Osteoblasts were incubated with viral supernatants for $6 \mathrm{~h}$ in the presence of $10 \mu \mathrm{g} / \mathrm{mL}$ polybrene (Sigma-Aldrich).

\section{Reverse transcription-polymerase chain reaction}

Total RNA was extracted from cultured cells using Qiazol lysis reagent (Qiagen, Hilden, Germany) according to the manufacturer's instructions, and $2 \mu \mathrm{g}$ of RNA was reverse transcribed into cDNA using SuperScript II Reverse Transcriptase (Life Technologies). To assess mRNA expression levels, the cDNA was amplified by polymerase chain reaction (PCR) using specific primers. The primer sequences were as follows: glyceraldehyde-3phosphate dehydrogenase (GAPDH), 5'-TGA CCA CAG TCC ATG CCA TCA CTG-3' and 5'-CAG GAG ACA ACC TGG TCC TCA GTG-3'; Crk, 5'-AGG CAG GGT AGT GGA GTG ATT CTC A-3' and 5'-CAT CTG TCA GCA AAC TGT CGA GCT AT-3'; CrkIII, 5'-AGG CAG GGT AGT GGA GTG ATT CTC A-3' and 5'-CGT TTC GCT GTA TCA GCA TTC CTA C-3'; and CrkL, 5'-GTG TCT CGC ACT ACA TCA TCA A-3' and 5'-GCT GAG ACA GAA CCC ACT GG-3'.

\section{Real-time PCR}

Quantitative real-time PCR analyses were performed in triplicate on a Rotor-Gene Q (Qiagen) using SYBR Green PCR Master Mix (Qiagen). Target gene expression was normalized to the level of an endogenous control, GAPDH. The relative quantitative value of each target gene compared with the calibrator for that target was expressed as $2^{-(\mathrm{Ct}-\mathrm{Cc})}$, where $\mathrm{Ct}$ and $\mathrm{Cc}$ represent the mean threshold cycle differences after normalization to GAPDH. The relative expression levels of the samples are presented in a semilog plot. The primer sequences were as follows: GAPDH, 5'-TGA CCA CAG TCC ATG CCA TCA CTG-3' and 5'-CAG GAG ACA ACC TGG TCC TCA GTG-3'; Crk, 5'-AGG CAG GGT AGT GGA GTG 
ATT CTC A-3' and 5'-CAT CTG TCA GCA AAC TGT CGA GCT AT-3'; Runx2, 5'-CCC AGC CAC CTT TAC CTA CA- $3^{\prime}$ and $5^{\prime}$-CAG CGT CAA CAC CAT CAT TC3'; ALP, 5'-CAA GGA TAT CGA CGT GAT CAT G-3' and 5'-GTC AGT CAG GTT GTT CCG ATT C-3'; BSP, 5'-GGA AGA GGA GAC TTC AAA CGA AG-3' and 5'CAT CCA CTT CTG CTT CTT CGT TC-3'; and Osteocalcin, 5'-ATG AGG ACC CTC TCT CTG CTG CTC AC-3' and 5'-AGA GCA AAC TGC AGA AGC TGA GAG-3'.

\section{Short interfering RNA (siRNA) transfection}

Control, Crk, and CrkL siRNAs were purchased from Dharmacon (Lafayette, CO) and transfected into osteoblasts using Lipofectamine 2000 (Life Technologies) according to the manufacturer's protocol.

\section{Western blotting}

Cultured cells were lysed in extraction buffer $[50 \mathrm{mM}$ Tris- $\mathrm{HCl}$ (pH 8.0), $150 \mathrm{mM} \mathrm{NaCl}, 1 \mathrm{mM}$ EDTA, $0.5 \%$ Nonidet P-40, $1 \mathrm{mM}$ PMSF, and protease inhibitor cocktail] and quantified using a BCA Protein Assay kit (Pierce, Rockford). Equal amounts of protein were subjected to sodium dodecyl sulfate-polyacrylamide gel electrophoresis and transferred to a polyvinylidene fluoride membrane (Millipore, Billerica, MA). Membranes were incubated with appropriate primary antibodies against Flag, actin (Sigma-Aldrich), Runx2 (Santa Cruz Biotechnology, Santa Cruz, CA), phospho-Smad, Smad, phospho-p38, p38, phospho-JNK, JNK, phospho-Erk, Erk (Cell Signaling Technology, Beverly, MA), and lamin B1 (Abchem, Cambridge, UK). Following washing and incubation with appropriate horseradish peroxidase-linked secondary antibodies, signals were detected with an LAS3000 luminescent image analyzer (GE Healthcare, Piscataway, NJ).

\section{Generation of Crkll transgenic mice}

Wild-type CrkII cDNA was fused to the mouse Col1 $\alpha 1$ promoter. Transgenic mice were generated by standard pronuclear injection into C57BL/6 mice. Genomic DNA was isolated from the tail, and mice were genotyped using PCR. The following primer sequences were used: 5'-GAG TTT TCG GAG ACG CTA AGC ACA TAG-3' and 5'GAG TTT TCG GAG ACG CTA AGC ACA TAG-3'.

\section{Microcomputed tomography analysis}

Distal femurs were isolated from 8-week-old wild type or CrkII transgenic mice, dissected, and fixed overnight in $75 \%$ ethanol. For microcomputed tomography $(\mu \mathrm{CT})$ analyses, the femurs were scanned using a high-resolution SkyScan 1172 system (SkyScan, Kontich, Belgium) at $50 \mathrm{kV}$ and $201 \mu \mathrm{A}$ with a $0.5 \mathrm{~mm}$ aluminum filter at a resolution of $11 \mu \mathrm{m}$ pixel $^{-1}$. Images were captured every $0.7^{\circ}$ over an angular range of $180^{\circ}$. Raw images were reconstructed into serial cross-sections, and femoral morphometric parameters were analyzed using image reconstruction software (NRecon 1.4; SkyScan), data analysis software (CTAn; SkyScan), and three-dimensional model visualization software (Ant 2.4; SkyScan).

\section{Bone histomorphometric analysis}

Tibiae were collected from 8-week-old wild-type or CrkII transgenic mice, fixed overnight in $4 \%$ paraformaldehyde, and decalcified in 5.5\% EDTA buffer for 2 weeks at $4{ }^{\circ} \mathrm{C}$. The decalcified tibiae were processed for paraffin embedding, and 4- $\mu \mathrm{m}$-thick longitudinal sections were prepared. Bone sections were deparaffinized, hydrated, and stained with hematoxylin and eosin (H\&E) or tartrate-resistant acid phosphatase.

\section{Statistical analysis}

All values are expressed as the mean \pm SD. Statistically significant differences were determined using a two-tailed Student's $t$-test for two independent samples. Differences with $p$ values less than 0.05 were considered statistically significant. Comparisons of three or more samples were analyzed by one-way analysis of variance.

\section{Results \\ Crkll negatively regulates osteoblast differentiation and function}

When we examined the expression levels of Crk family members in osteoblasts, all Crk family members, including CrkI, CrkII, CrkIII, and CrkL, were expressed continuously during osteoblast differentiation (Fig. S1). To determine the role of CrkII in osteoblast differentiation and function, control- or CrkII-transduced osteoblasts were cultured in OGM, and the ALP activity and alizarin red staining intensities were then quantified. ALP activity was strongly induced by OGM stimulation, and this induction was greatly reduced in CrkII-overexpressing cells (Fig. 1a). Similarly, bone mineralization measured by quantifying the alizarin red staining intensity was also inhibited in CrkII-overexpressing cells compared with that in control cells (Fig. 1b). Consistent with changes in ALP activity and bone mineralization, the expression of the typical osteogenic marker genes Runx2, Alpl, Ibsp, and Bglap was significantly inhibited by CrkII overexpression (Fig. 1c).

To confirm the role of CrkII in osteoblast differentiation and function, osteoblasts were transfected with siRNAs targeting CrkII. Downregulation of endogenous CrkII expression (Fig. 2a) resulted in increased ALP activity, bone mineralization, and expression of typical osteogenic marker genes (Fig. 2b-d). Collectively, these data suggest that CrkII negatively regulates osteoblast differentiation and function. 
a

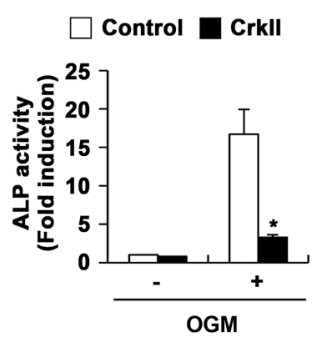

b

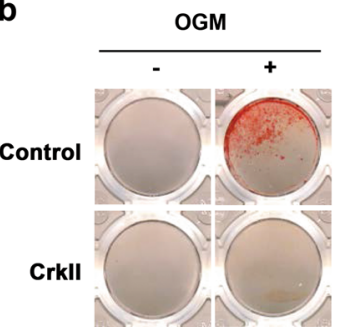

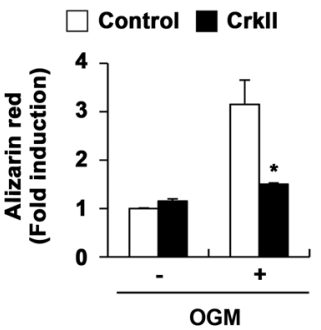

C

$\square$ Control $\square$ Crkll
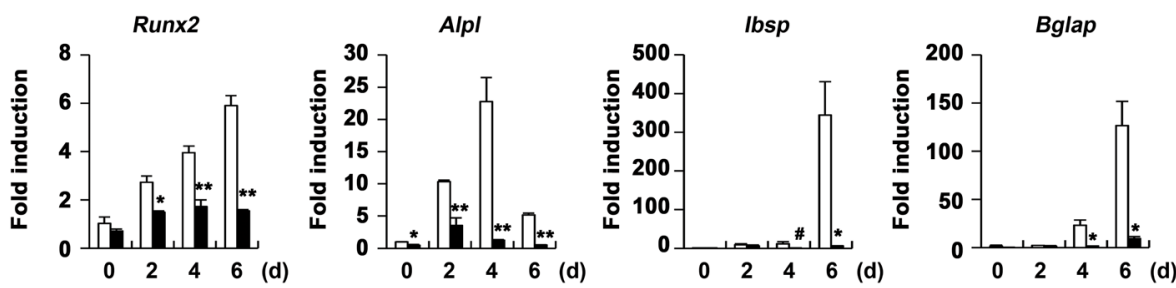

Fig. 1 Crkll overexpression inhibits osteoblast differentiation and function. a-c Primary osteoblast precursor cells were transduced with pMXIRES-EGFP (control) or Crkll retrovirus and cultured in OGM containing BMP2, ascorbic acid, and $\beta$-glycerophosphate. a Cells were cultured for 3 days and subjected to an ALP activity assay. b Cells were cultured for 9 days, fixed, and stained with alizarin red (left panel). Staining intensities were quantified at $562 \mathrm{~nm}$ via densitometry (right panel). c Total RNA was collected at each indicated time point, and real-time PCR was performed to evaluate the expression of the target genes. Data are expressed as the mean \pm SD of triplicate samples. ${ }^{*} p<0.05,{ }^{*} p<0.01,{ }^{* *} p<0.001$ versus control
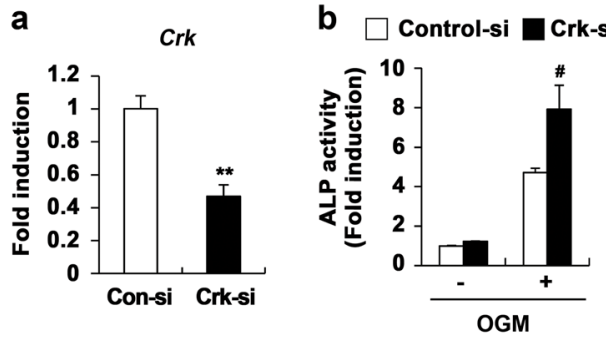

d
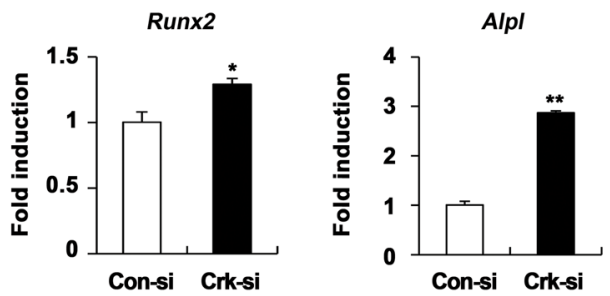
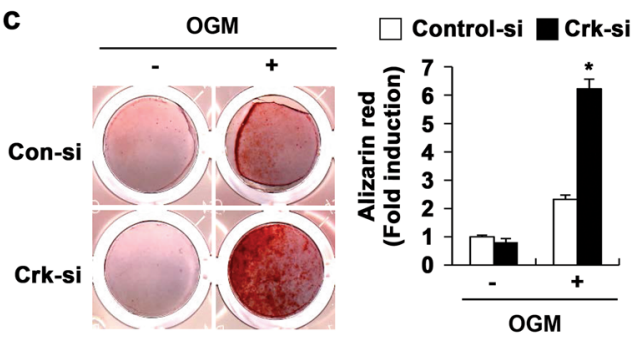

Fig. 2 Crkll downregulation enhances osteoblast differentiation and function. a Control siRNA- or Crk siRNA-transfected osteoblasts were cultured for 2 days. Real-time PCR was performed to assess Crkll mRNA expression. b-d Osteoblasts were transfected with control siRNA or Crk siRNA and cultured in OGM. b Cells were cultured for 3 days and subjected to an ALP activity assay. c Cells were cultured for 9 days, fixed, and stained with alizarin red (left panel). Staining intensities were quantified at $562 \mathrm{~nm}$ via densitometry (right panel). d Total RNA was collected at each indicated time point, and real-time PCR was performed to evaluate the expression of the target genes. Data are expressed as the mean \pm SD of triplicate samples. ${ }^{\#} p<0.05,{ }^{*} p<0.01,{ }^{* *} p<0.001$ versus control

Crkll and CrkL have overlapping functions in osteoblasts

Since all Crk family adaptor proteins were expressed in osteoblasts (Fig. S1), we investigated the roles of other Crk family adaptors in osteoblasts. As shown in Fig. 3a, b, similar to the effects of CrkII overexpression, the overexpression of only CrkL significantly inhibited
ALP activity and bone mineralization. CrkL overexpression also reduced the expression of typical osteogenic marker genes (Fig. S2). Furthermore, downregulation of both CrkII and CrkL synergistically increased bone mineralization (Fig. S3). These results indicated that among the Crk family adaptors, CrkII 

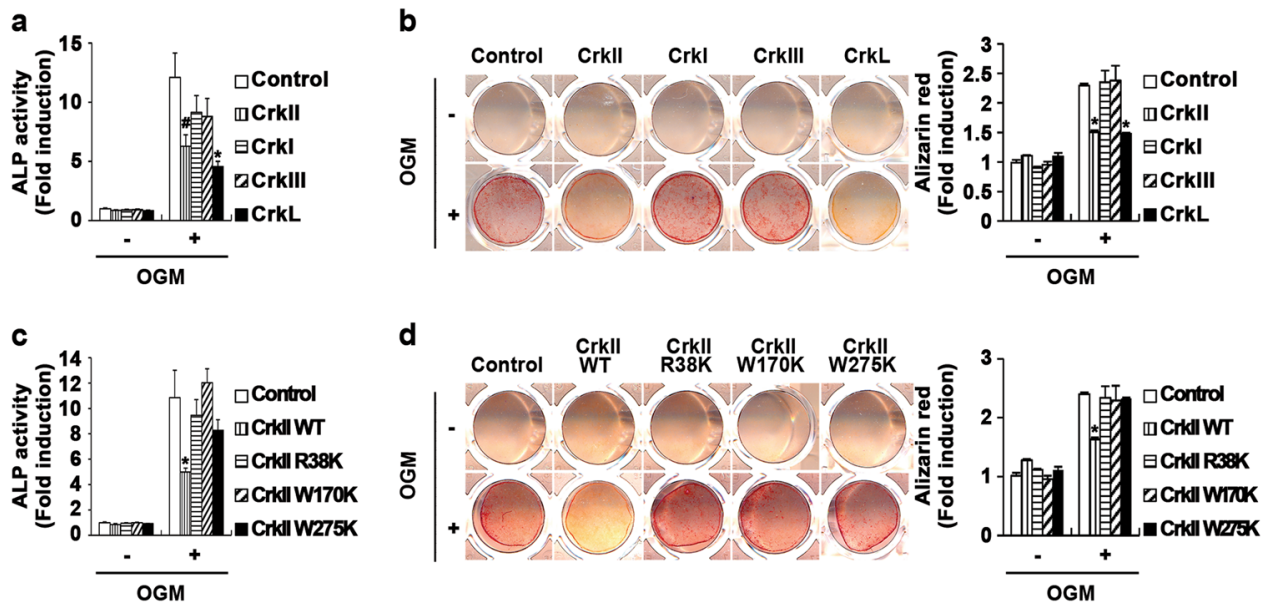

Fig. 3 The roles of the three SH domains in CrkIl in osteoblasts. a-b Osteoblasts were transduced with pMX-IRES-EGFP (control), Crkll, Crkl, Crklll, or CrkL retrovirus. Transduced osteoblasts were cultured in OGM. a Cells were cultured for 3 days and subjected to an ALP activity assay. b Cells were cultured for 9 days, after which they were fixed and stained with alizarin red (left panel). Staining intensities were quantified at $562 \mathrm{~nm}$ via densitometry (right panel). c-d Osteoblasts were transduced with pMX-IRES-EGFP (control), Crkll-WT, Crkll-R38K, Crkll-W170K, or Crkll-W275K retrovirus. Transduced osteoblasts were cultured in OGM. c Cells were cultured for 3 days and subjected to an ALP activity assay. d Cells were cultured for 9 days, after which they were fixed and stained with alizarin red (left panel). Staining intensities were quantified at $562 \mathrm{~nm}$ via densitometry (right panel). Data are expressed as the mean \pm SD of triplicate samples. ${ }^{*} p<0.05,{ }^{*} p<0.01$ versus control

and CrkL, but not CrkI and CrkIII, inhibit osteoblast differentiation and function.

Although CrkL is the product of a distinct gene, it contains one $\mathrm{SH} 2$ domain and two $\mathrm{SH} 3$ domains like CrkII, whereas CrkI lacks the cSH3 domain and regulatory tyrosine phosphorylation site, and CrkIII contains a partially disrupted $\mathrm{cSH} 3$ domain $^{24,25,30-32}$. Therefore, we tested whether three $\mathrm{SH}$ domains are required for the function of CrkII in osteoblasts. Overexpression of CrkII significantly inhibited ALP activity and bone mineralization, but overexpression of R38K CrkII (mutated in SH2), W170K CrkII (mutated in nSH3), and W275K CrkII (mutated in $\mathrm{CSH} 3$ ) did not inhibit ALP activity or bone mineralization (Fig. 3c, d). These data suggest that the three SH domains in CrkII are required for CrkIIregulated osteoblast differentiation and function.

\section{Crkll regulates osteoblast differentiation and function via JNK activation}

Next, we determined which signaling pathway is most responsible for the CrkII-induced inhibition of osteoblast differentiation and function. As shown in Fig. 4, BMP2 stimulation successfully induced the phosphorylation of Smad1/5/8, Erk, and p38, which positively regulate osteoblast differentiation ${ }^{33-37}$. When CrkII was overexpressed in osteoblasts, the BMP2-induced phosphorylation of p38 was slightly increased, but that of Smad1/5/8 and Erk was not. Interestingly, in control cells, BMP2 did not induce the phosphorylation of JNK, which negatively regulates osteoblast differentiation ${ }^{6}$, whereas the phosphorylation of JNK was increased in

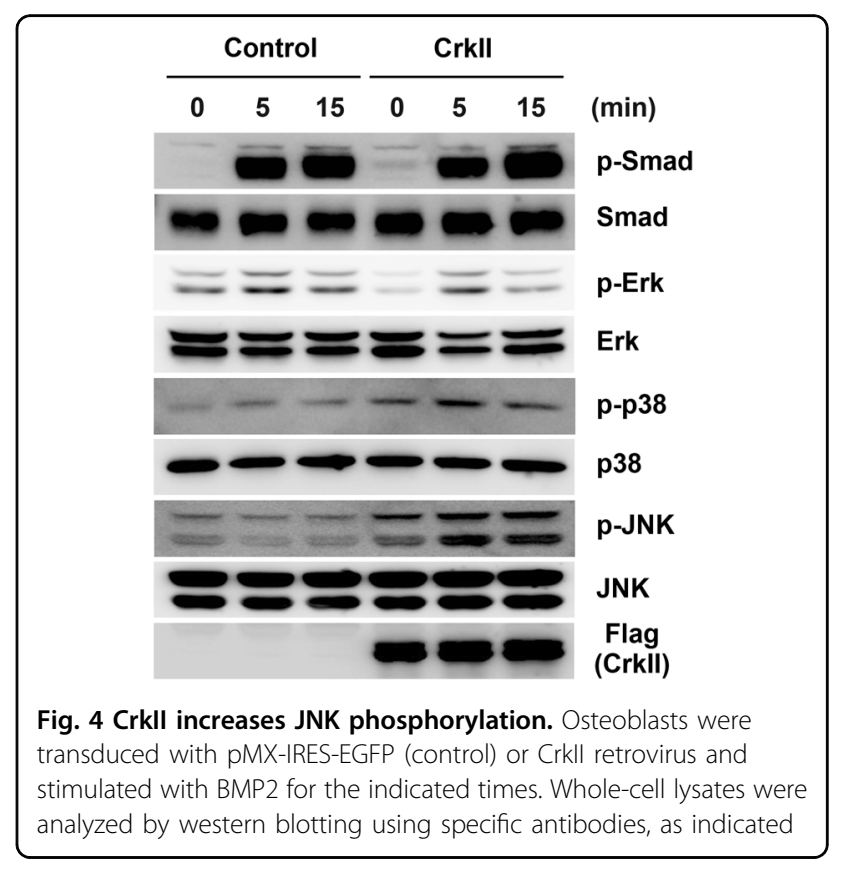

CrkII-overexpressing cells and even more strongly increased by BMP2 stimulation (Fig. 4).

To examine the correlation between reduced osteoblast differentiation and increased p38 phosphorylation by CrkII, we used a specific p38 MAPK inhibitor, SB203580. As shown in Fig. S4, treatment with SB203580 did not affect the CrkII-induced reduction in ALP activity, indicating that CrkII regulates osteoblast differentiation and function through signaling pathways other than the $\mathrm{p} 38$ 

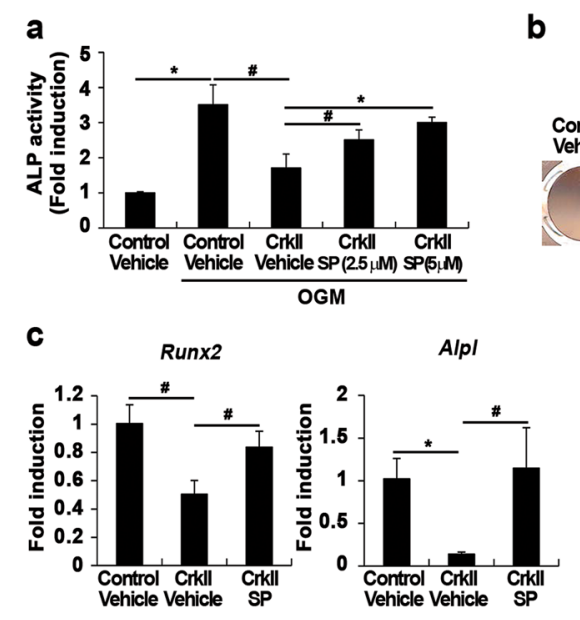

b
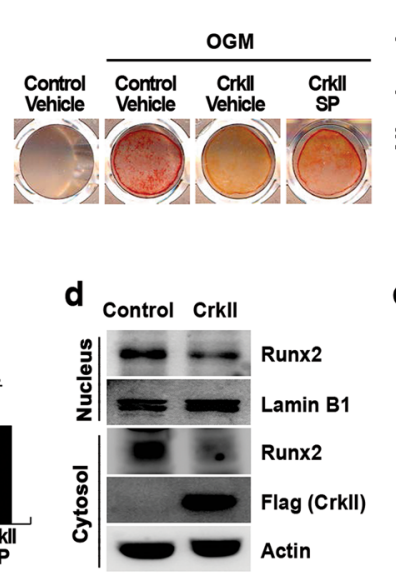

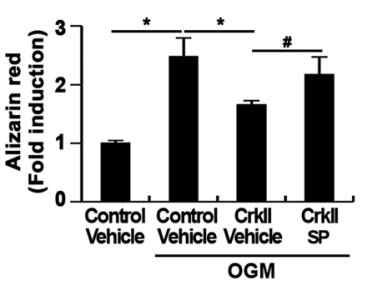

Control Crkll

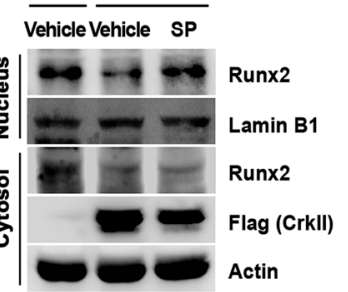

Fig. 5 Crkll regulates osteoblast differentiation through enhancing JNK activation. a-e Osteoblasts were transduced with pMX-IRES-EGFP (control) or Crkll retrovirus. Transduced osteoblasts were treated with vehicle or SP600125 (2.5 or $5 \mu \mathrm{M}$ ) and cultured in OGM. a Cells were cultured for 3 days and subjected to an ALP activity assay. b Cells were cultured for 9 days, after which they were fixed and stained with alizarin red (left panel). Staining intensities were quantified at $562 \mathrm{~nm}$ via densitometry (right panel). c Total RNA was collected from cells cultured for 4 days, and real-time PCR was performed to evaluate expression of the target genes. $\mathbf{d}$, e Cytoplasmic and nuclear fractions were harvested from cultured cells and subjected to western blot analysis using specific antibodies, as indicated. Data are expressed as the mean \pm SD of triplicate samples. ${ }^{\#} p<0.05$, ${ }^{*} p<0.01$ versus control

MAPK pathway. Next, we tested whether CrkII inhibits osteoblast differentiation and function through activation of the JNK pathway. Treatment with a specific JNK inhibitor, SP600125, increased ALP activity, bone mineralization, and the expression of osteogenic marker genes reduced by CrkII overexpression (Fig. $5 \mathrm{a}-\mathrm{c}$ ). Therefore, these results indicated that CrkII regulates osteoblast differentiation and function through enhancing activation of the JNK pathway. To further examine the correlation between reduced osteoblast differentiation and increased JNK activation by CrkII, we investigated the effects of CrkII and SP600125 on Runx2 translocation. Importantly, CrkII inhibited the nuclear translocation of Runx2, and its effects were attenuated by the inhibition of JNK activity (Fig. 5d, e). Collectively, these results indicate that activation of the JNK pathway by CrkII reduces osteoblast differentiation and function by inhibiting the nuclear translocation of Runx2.

We previously reported that the role of CrkII in osteoclast differentiation and function is linked to Rac1 activation $^{23}$. Moreover, BMP2-induced Rac1 activation has been shown to inhibit osteoblast differentiation ${ }^{38}$. Therefore, we examined whether the negative role of CrkII in osteoblast differentiation is associated with Rac1 activation. Treatment with a specific Rac1 inhibitor, NSC23766, increased ALP activity, bone mineralization, and expression of osteogenic marker genes reduced by CrkII overexpression (Fig. 6a-c), indicating that the negative role of CrkII in osteoblast differentiation is linked to Rac1 activation.
Transgenic mice overexpressing Crkll in osteoblasts exhibit low bone mass

After the inhibitory roles of CrkII in osteoblast differentiation and function were confirmed, to further investigate the effects of CrkII on bone mass in vivo, we generated transgenic mice expressing CrkII under the control of the alpha-1 type I collagen promoter. As shown in Fig. $7 \mathrm{a}, \mu \mathrm{CT}$ analysis revealed a significant decrease in bone mass in CrkII transgenic mice compared with that in their wild-type littermates. The trabecular bone volume, thickness, and number were significantly decreased, whereas trabecular bone separation was significantly increased in CrkII transgenic mice compared with those in wild-type mice (Fig. 7b). In addition, the number of osteoblasts per bone perimeter was decreased in CrkII transgenic mice (Fig. 7c, d). Therefore, these results indicated that CrkII negatively regulates osteoblast differentiation and function in vivo.

\section{Discussion}

Bone homeostasis is maintained by a constant balance between the activity of bone-resorbing osteoclasts and bone-forming osteoblasts. An imbalance between old bone resorption and new bone formation results in abnormal bone remodeling and the development of bone diseases, such as osteoporosis, Paget's disease, and rheumatoid arthritis ${ }^{39}$. Many drugs and agents that either inhibit bone resorption or promote bone formation have been proposed for the treatment of bone diseases. Because bone remodeling is a coupled process between bone 

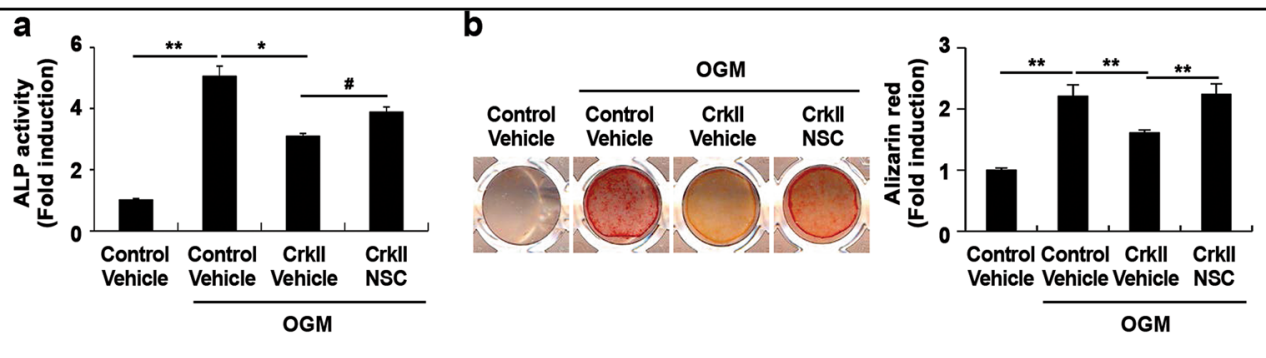

C
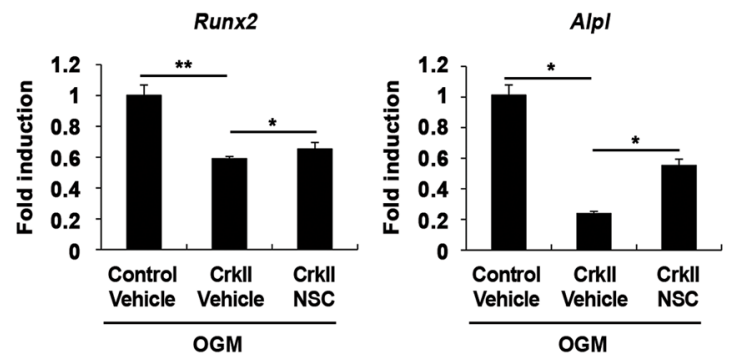

Fig. 6 Crkll regulates osteoblast differentiation via Rac1 activation. a-c Osteoblasts were transduced with pMX-IRES-EGFP (Control) or Crkll retrovirus. Transduced osteoblasts were treated with vehicle or NSC23766 (50 $\mu \mathrm{M})$ and cultured in OGM. a Cells were cultured for 3 days and subjected to an ALP activity assay. b Cells were cultured for 9 days, after which they were fixed and stained with alizarin red (left panel). Staining intensities were quantified at $562 \mathrm{~nm}$ via densitometry (right panel). c Total RNA was collected from cells cultured for 4 days, and real-time PCR was performed to evaluate expression of the target genes. Data are expressed as the mean \pm SD of triplicate samples. ${ }^{*} p<0.05,{ }^{*} p<0.01$, ${ }^{* *} p<0.001$ versus control

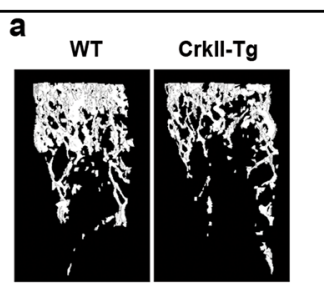

C

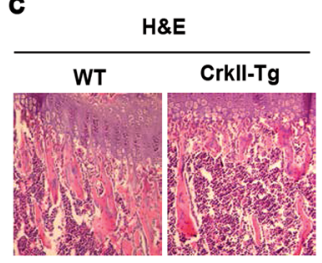

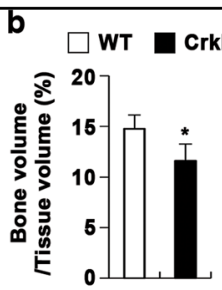
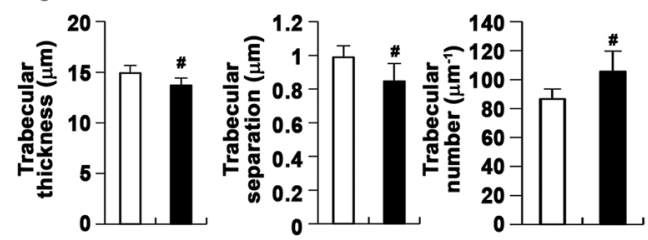

d

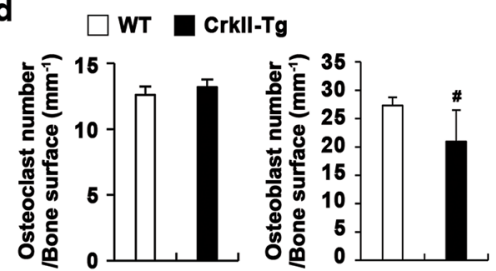

Fig. 7 Crkll transgenic mice exhibit low bone mass. a Representative three-dimensional images of the femurs from Crkll transgenic mice and their wild-type littermates. b Bone volume to tissue volume and the trabecular bone thickness, separation, and number were assessed using $\mu C T$ measurements ( $n=7$ or 5). $\mathbf{c}$ H\&E and TRAP staining of histological sections of proximal tibiae. $\mathbf{d}$ The numbers of osteoblasts and osteoclasts per bone surface area were assessed ( $n=5$ or 4 ). ${ }^{*} p<0.05,{ }^{*} p<0.01$ versus control

resorption and bone formation, there are certain limitations regarding drugs and agents targeting only one type of bone cell (osteoclast or osteoblast). For example, bisphosphonates, which are typical antiresorptive agents, effectively inhibit bone resorption but simultaneously inhibit bone formation, while parathyroid hormone, an anabolic agent, increases bone formation but also increases bone resorption ${ }^{40-43}$. Although our previous study showed that CrkII plays an important role in osteoclast differentiation and function, the role of CrkII in osteoblast differentiation and function remains unknown. To determine whether CrkII can simultaneously regulate bone resorption and bone formation, in the present study, we investigated the role of CrkII in osteoblast differentiation and function.

Our results evidently showed the negative role of CrkII in osteoblast differentiation and function. Overexpression of CrkII inhibited ALP activity, bone mineralization, and 
the expression of osteogenic marker genes, whereas downregulation of CrkII expression increased these factors. Interestingly, CrkII promoted JNK activation in osteoblasts, and inhibition of JNK activity by a JNK inhibitor completely reversed the inhibitory effects of CrkII on ALP activity, bone mineralization, and expression of osteogenic marker genes. Taken together, these results suggest that CrkII plays a negative role in osteoblast differentiation and function via the activation of JNK.

Although JNK is activated during osteoblast differentiation, its exact role in osteoblasts is somewhat controversial. JNK2 activation during osteoblast differentiation has been reported to be essential for latestage differentiation in preosteoblast cells ${ }^{44}$. In contrast, activation of JNK has been shown to contribute to the inhibitory effects of tumor necrosis factor- $\alpha$ or TGF- $\beta$ on osteoblast differentiation ${ }^{45-47}$. Notably, Huang et al. ${ }^{6}$ recently reported that JNK1 negatively regulates osteoblast differentiation via the phosphorylation of Runx2 on Ser104. That CrkII overexpression strongly phosphorylated JNK in osteoblasts and the blockade of JNK activation enhanced osteoblast differentiation and function inhibited by CrkII, even though the JNK pathway is strongly activated during osteoblast differentiation, suggests that activated JNK acts as an inhibitory factor for osteoblast differentiation.

Several studies have reported that Crk connects multiple cellular stimuli to the JNK signaling pathway. The JNK pathway was previously shown to be activated in $\mathrm{v}$-Crktransformed cells and cells transiently overexpressing $\mathrm{CrkII}^{48,49}$. Moreover, a direct interaction between JNK1 and CrkII was shown to be critical for JNK activation ${ }^{50}$. We found that CrkII connects osteogenic stimuli to the JNK pathway probably through binding with JNK1 (data not shown). Although CrkII phosphorylated both JNK1 and JNK2 in osteoblasts, its effect on the activation of JNK1 was stronger than that on the activation of JNK2. Huang et al. ${ }^{6}$ showed that JNK1 activation was strongly increased after BMP2 stimulation in multipotent C2C12 cells and preosteoblast MC3T3 cells, whereas JNK2 was activated to a lesser extent by BMP2 than JNK1. Furthermore, the CrkII-binding domain is conserved in JNK1 but not JNK2, and CrkII interacts only weakly with $\mathrm{JNK} 2^{50}$. Collectively, these data support the possibility that the negative role of CrkII in osteoblast differentiation and function is linked to the activation of JNK1 rather than JNK2 via the direct interaction of CrkII with JNK1.

We showed here that the three SH domains in CrkII are required for CrkII-mediated osteoblast differentiation and function. Although the interaction between CrkII and JNK1 occurs through the N-terminal SH3 domain of CrkII, interestingly, JNK1 activation is significantly reduced by CrkII mutated in its $\mathrm{SH} 2$ domain compared to its activation by wild-type CrkII ${ }^{50}$. Since CrkII lacks a kinase domain, the optimal activation of JNK1 by CrkII likely requires the recruitment of several kinases, which may occur through the $\mathrm{SH} 2$ domain or C-terminal $\mathrm{SH} 3$ domain of CrkII, and the direct binding of CrkII with JNK1. For example, p130Cas, an adaptor protein containing several phosphorylatable tyrosine residues, is involved in JNK activation through the interaction of p130Cas with the SH2 domain of CrkII ${ }^{50}$. Since the present data strongly suggest that the function of CrkII in osteoblasts is linked to JNK activation, other domains in CrkII besides the $\mathrm{N}$-terminal $\mathrm{SH} 3$ domain might contribute to JNK activation to regulate osteoblast differentiation and function.

We have previously reported that the role of CrkII in osteoclasts is linked to Rac1 activation ${ }^{23}$. Inhibition of Rac1 was shown to promote osteoblast differentiation upon BMP2 stimulation ${ }^{38}$. In the present study, we investigated whether the function of CrkII in osteoblasts is correlated with Rac1 activation, as it is in osteoclasts. Treatment with a Rac1 inhibitor partially reversed the inhibitory effects of CrkII on osteoblast differentiation, including its effects on ALP activity and the expression of Runx 2 and ALP. However, the effects of the simultaneous inhibition of JNK and Rac1 were not synergistic, most likely due to the cytotoxic effects of pharmacological inhibitors (data not shown). Moreover, the inhibition of Rac1 did not affect JNK activation in osteoblasts overexpressing CrkII (data not shown). These results collectively suggest that Rac1 participates in CrkII-regulated osteoblast differentiation independent of the JNK signaling pathway. However, since several lines of evidence suggest that Rac1 is essential for CrkII-induced JNK activation $^{49}$, we cannot exclude the possibility that Rac1 function in osteoblasts is closely related to JNK activation. Constitutively active Rac1 readily causes JNK activation, whereas a dominant negative form of Rac (Rac1N17) completely blocked Crk-induced JNK activation ${ }^{49}$. Collectively, these results suggest that Rac1 activation is a necessary step connecting Crk to JNK activation. Therefore, it is possible that the Rac1 inhibitor used in our experiments did not completely block Rac1 activity or that JNK was activated through the Ras pathway, an alternative pathway involving Crk-induced JNK activation, rather than the Rac1 pathway. It is necessary to identify whether Rac1 is involved in connecting CrkII to JNK activation in osteoblasts. Our future studies will investigate this issue.

In summary, our results showed that CrkII negatively regulates osteoblast differentiation and function through JNK activation, which reduces the expression of Runx2. Since CrkII also acts as a positive regulator of osteoclast differentiation and function, CrkII can regulate bone resorption as well as bone formation. Therefore, CrkII 
may serve as a new therapeutic target for the treatment of bone diseases by increasing bone formation and simultaneously inhibiting bone resorption.

\section{Acknowledgements}

This research was supported by grants from the Basic Science Research Program (2019R1A5A2027521 and 2015R1C1A1A02037671) through the National Research Foundation of Korea (NRF), which is funded by the Ministry of Science, ICT, and Future Planning.

\section{Author details}

'Department of Pharmacology, Chonnam National University Medical School, Gwangju 61469, Republic of Korea. ${ }^{2}$ Department of Biomedical Sciences, Chonnam National University Medical School, Gwangju 61469, Republic of Korea. ${ }^{3}$ Department of Anatomy, Chonnam National University Medical School, Gwangju 61469, Republic of Korea

\section{Conflict of interest}

The authors declare that they have no conflict of interest.

\section{Publisher's note}

Springer Nature remains neutral with regard to jurisdictional claims in published maps and institutional affiliations.

Supplementary information accompanies this paper at https://doi.org/ 10.1038/s12276-019-0314-3.

Received: 13 February 2019 Revised: 1 July 2019 Accepted: 5 July 2019. Published online: 25 September 2019

\section{References}

1. Choi, Y. H. et al. Src enhances osteogenic differentiation through phosphorylation of Osterix. Mol. Cell. Endocrinol. 407, 85-97 (2015).

2. Kawamura, N. et al. Akt1 in osteoblasts and osteoclasts controls bone remodeling. PLOS ONE 2, e1058 (2007).

3. Komori, T. Regulation of osteoblast differentiation by transcription factors J. Cell. Biochem. 99, 1233-1239 (2006).

4. Lee, M. H. et al. Transient upregulation of CBFA1 in response to bone morphogenetic protein-2 and transforming growth factor beta1 in C2C12 myogenic cells coincides with suppression of the myogenic phenotype but is not sufficient for osteoblast differentiation. J. Cell. Biochem. 73, 114-125 (1999).

5. Lee, K. S. et al. Run $\times 2$ is a common target of transforming growth factor beta1 and bone morphogenetic protein 2, and cooperation between Runx2 and Smad5 induces osteoblast-specific gene expression in the pluripotent mesenchymal precursor cell line C2C12. Mol. Cell. Biol. 20 8783-8792 (2000).

6. Huang, Y. F., Lin, J. J., Lin, C. H., Su, Y. \& Hung, S. C. c-Jun N-terminal kinase 1 negatively regulates osteoblastic differentiation induced by BMP2 via phosphorylation of Runx2 at Ser104. J. Bone Miner. Res. 27, 1093-1105 (2012).

7. Blum, B., Moseley, J., Miller, L., Richelsoph, K. \& Haggard, W. Measurement of bone morphogenetic proteins and other growth factors in demineralized bone matrix. Orthopedics 27, s161-s165 (2004).

8. Matsubara, T. et al. BMP2 regulates Osterix through Ms×2 and Runx2 during osteoblast differentiation. J. Biol. Chem. 283, 29119-29125 (2008).

9. Ulsamer, A. et al. BMP-2 induces Osterix expression through up-regulation of Dlx5 and its phosphorylation by p38. J. Biol. Chem. 283, 3816-3826 (2008).

10. Yamaguchi, A., Komori, T. \& Suda, T. Regulation of osteoblast differentiation mediated by bone morphogenetic proteins, hedgehogs, and Cbfa1. Endocr. Rev. 21, 393-411 (2000).

11. Liu, T. M. \& Lee, E. H. Transcriptional regulatory cascades in Runx2-dependent bone development. Tissue Eng. Part B Rev. 19, 254-263 (2013).

12. Phillips, J. E., Hutmacher, D. W., Guldberg, R. E. \& Garcia, A. J. Mineralization capacity of Runx2/Cbfa1-genetically engineered fibroblasts is scaffold dependent. Biomaterials 27, 5535-5545 (2006).

13. Otto, F. et al. Cbfa1, a candidate gene for cleidocranial dysplasia syndrome, is essential for osteoblast differentiation and bone development. Cell 89 , 765-771 (1997)
14. Komori, T. et al. Targeted disruption of Cbfa1 results in a complete lack of bone formation owing to maturational arrest of osteoblasts. Cell 89, 755-764 (1997)

15. Mundlos, S. et al. Mutations involving the transcription factor CBFA1 cause cleidocranial dysplasia. Cell 89, 773-779 (1997).

16. Ducy, P., Zhang, R., Geoffroy, V., Ridall, A. L. \& Karsenty, G. Osf2/Cbfa1: a transcriptional activator of osteoblast differentiation. Cell 89, 747-754 (1997).

17. Massague, J., Blain, S. W. \& Lo, R. S. TGFbeta signaling in growth control, cancer, and heritable disorders. Cell 103, 295-309 (2000).

18. Xiao, G. et al. Bone morphogenetic proteins, extracellular matrix, and mitogenactivated protein kinase signaling pathways are required for osteoblastspecific gene expression and differentiation in MC3T3-E1 cells. J. Bone Miner. Res. 17, 101-110 (2002).

19. Nohe, A. et al. The mode of bone morphogenetic protein (BMP) receptor oligomerization determines different BMP-2 signaling pathways. J. Biol. Chem. 277, 5330-5338 (2002).

20. Guo, X. \& Wang, X. F. Signaling cross-talk between TGF-beta/BMP and other pathways. Cell Res. 19, 71-88 (2009).

21. Ge, C. et al. Identification and functional characterization of ERK/MAPK phosphorylation sites in the Runx2 transcription factor. J. Biol. Chem. 284, 32533-32543 (2009).

22. Greenblatt, M. B. et al. Thep38 MAPK pathway is essential for skeletogenesis and bone homeostasis in mice. J. Clin. Invest. 120, 2457-2473 (2010).

23. Kim, J. H. et al. Role of Crkll signaling in RANKL-induced osteoclast differentiation and function. J. Immunol. 196, 1123-1131 (2016).

24. Matsuda, M. et al. Two species of human CRK CDNA encode proteins with distinct biological activities. Mol. Cell. Biol. 12, 3482-3489 (1992).

25. Reichman, C. T., Mayer, B. J., Keshav, S. \& Hanafusa, H. The product of the cellular crk gene consists primarily of $\mathrm{SH} 2$ and $\mathrm{SH} 3$ regions. Cell. Growth Differ. 3, 451-460 (1992).

26. Klemke, R. L. et al. CAS/Crk coupling serves as a "molecular switch" for induction of cell migration. J. Cell Biol. 140, 961-972 (1998).

27. Nakashima, N. et al. The functional role of $\mathrm{Crkll}$ in actin cytoskeleton organization and mitogenesis. J. Biol. Chem. 274, 3001-3008 (1999).

28. Cho, S. Y. \& Klemke, R. L. Extracellular-regulated kinase activation and CAS/Crk coupling regulate cell migration and suppress apoptosis during invasion of the extracellular matrix. J. Cell Biol. 149, 223-236 (2000).

29. Gumienny, T. L. et al. CED-12/ELMO, a novel member of the Crkll/Dock180/Rac pathway, is required for phagocytosis and cell migration. Cell 107, 27-41 (2001).

30. Feller, S. M. Crk family adaptors-signalling complex formation and biological roles. Oncogene 20, 6348-6371 (2001).

31. Prosser, S., Sorokina, E., Pratt, P. \& Sorokin, A. Crklll: a novel and biologically distinct member of the Crk family of adaptor proteins. Oncogene 22, 4799-4806 (2003).

32. ten Hoeve, J., Morris, C., Heisterkamp, N. \& Groffen, J. Isolation and chromosomal localization of CRKL, a human crk-like gene. Oncogene 8, 2469-2474 (1993).

33. Xiao, G. et al. MAPK pathways activate and phosphorylate the osteoblastspecific transcription factor, Cbfa1. J. Biol. Chem. 275, 4453-4459 (2000).

34. Franceschi, R. T. et al. Multiple signaling pathways converge on the Cbfa1/ Runx2 transcription factor to regulate osteoblast differentiation. Connect. Tissue Res. 44(Suppl 1), 109-116 (2003).

35. Lee, K. S., Hong, S. H. \& Bae, S. C. Both the Smad and p38 MAPK pathways play a crucial role in Runx2 expression following induction by transforming growth factor-beta and bone morphogenetic protein. Oncogene 21, 7156-7163 (2002).

36. Hu, Y., Chan, E., Wang, S. X. \& Li, B. Activation of p38 mitogen-activated protein kinase is required for osteoblast differentiation. Endocrinology 144, 2068-2074 (2003).

37. Jun, J. H. et al. BMP2-activated Erk/MAP kinase stabilizes Runx2 by increasing p300 levels and histone acetyltransferase activity. J. Biol. Chem. 285 36410-36419 (2010).

38. Onishi, M., Fujita, Y., Yoshikawa, H. \& Yamashita, T. Inhibition of Rac1 promotes BMP-2-induced osteoblastic differentiation. Cell. Death Dis. 4, e698 (2013).

39. Feng, X. \& McDonald, J. M. Disorders of bone remodeling. Annu. Rev. Pathol. 6 121-145 (2011)

40. Liu, S. et al. Bovine parathyroid hormone enhances osteoclast bone resorption by modulating V-ATPase through PTH1R. Int. J. Mol. Med. 37, 284-292 (2016).

41. Zhao, W., Byrne, M. H., Boyce, B. F. \& Krane, S. M. Bone resorption induced by parathyroid hormone is strikingly diminished in collagenase-resistant mutant mice. J. Clin. Invest. 103, 517-524 (1999). 
42. Lane, N. E. \& Kelman, A. A review of anabolic therapies for osteoporosis. Arthritis Res. Ther. 5, 214-222 (2003).

43. Pazianas, M., van der Geest, S. \& Miller, P. Bisphosphonates and bone quality. Bonekey. Rep. 3, 529 (2014)

44. Matsuguchi, T. et al. JNK activity is essential for Atf4 expression and late-stage osteoblast differentiation. J. Bone Miner. Res. 24, 398-410 (2009).

45. Mukai, T. et al. TNF-alpha inhibits BMP-induced osteoblast differentiation through activating SAPKJJNK signaling. Biochem. Biophys. Res. Commun. 356, 1004-1010 (2007).

46. Maeda, S., Hayashi, M., Komiya, S., Imamura, T. \& Miyazono, K. Endogenous TGF-beta signaling suppresses maturation of osteoblastic mesenchymal cells. EMBO J. 23, 552-563 (2004).
47. Sowa, H., Kaji, H., Yamaguchi, T., Sugimoto, T. \& Chihara, K. Activations of ERK1/ 2 and JNK by transforming growth factor beta negatively regulate Smad3induced alkaline phosphatase activity and mineralization in mouse osteoblastic cells. J. Biol. Chem. 277, 36024-36031 (2002).

48. Tanaka, S., Ouchi, T. \& Hanafusa, H. Downstream of Crk adaptor signaling pathway: activation of Jun kinase by $v$-Crk through the guanine nucleotide exchange protein C3G. Proc. Natl Acad. Sci. USA 94, 2356-2361 (1997).

49. Dolfi, F. et al. The adaptor protein Crk connects multiple cellular stimuli to the JNK signaling pathway. Proc. Natl Acad. Sci. USA 95, 15394-15399 (1998).

50. Girardin, S. E. \& Yaniv, M. A direct interaction between JNK1 and Crkll is critical for Rac1-induced JNK activation. EMBO J. 20, 3437-3446 (2001). 